To appear in the Astronomical Journal April 2006

\title{
M33's Variable A - A Hypergiant Star More Than 35 Years in Eruption
}

\author{
Roberta M. Humphreys, Terry J. Jones, Elisha Polomski, Michael Koppelman, Andrew \\ Helton, Kristen McQuinn, Robert D. Gehrz, C. E. Woodward \\ School of Physics and Astronomy, University of Minnesota, Minneapolis, MN 55455 \\ roberta@aps . umn. edu \\ R. Mark Wagner, Karl Gordon, Joannah Hinz \\ Steward Observatory, University of Arizona, Tucson, AZ 85721 \\ and \\ S. P. Willner \\ Harvard-Smithsonian Center for Astrophysics, Cambridge, MA 02138
}

\begin{abstract}
Variable A in M33 is a member of a rare class of highly luminous, evolved stars near the upper luminosity boundary that show sudden and dramatic shifts in apparent temperature due to the formation of optically thick winds in high mass loss episodes. Recent optical and infrared spectroscopy and imaging reveal that its "eruption" begun in $\sim 1950$ has ended, lasting $\approx 45$ yrs. Our current observations show major changes in its wind from a cool, dense envelope to a much warmer state surrounded by low density gas with rare emission lines of Ca II, [Ca II] and K I. Its spectral energy distribution has unexpectedly changed, especially at the long wavelengths, with a significant decrease in its apparent flux, while the star remains optically obscured. We conclude that much of its radiation is now escaping out of our line of sight. We attribute this to the changing structure and distribution of its circumstellar ejecta corresponding to the altered state of its wind as the star recovers from a high mass loss event.
\end{abstract}

Subject headings: stars:supergiants, stars:winds, stars:individual(M33 Var A) 


\section{Introduction}

Variable A in M33 is one of the highly luminous and unstable stars that define the upper luminosity limit in the Hertzsprung-Russell Diagram for evolved cool stars (see Humphreys and Davidson 1994). It was one of the original Hubble - Sandage variables (Hubble and Sandage 1953) and at its maximum light in 1950 one of the visually brightest stars in M33. Its historical light curve shows a rapid decline from maximum by more than three magnitudes in less than a year followed by a brief recovery and a second decline. It had an intermediate F-type spectrum at maximum consistent with its observed colors. After its second decline to fainter than 18th magnitude (see light curves in Hubble and Sandage 1953 and Rosino and Bianchini 1973), no further observations were obtained until our optical and near infrared photometry beginning in 1977. In 1985-86 Var A had the spectrum of an M-type supergiant (Humphreys, Jones and Gehrz 1987, hereafter HJG), and its large infrared excess at $10 \mu \mathrm{m}$ and spectral energy distribution showed that it was still as luminous $\left(5 \times 10^{5} L_{\odot}\right)$ as it was at its maximum light in the visible. Thus its large photometric and spectral variations had occurred at nearly constant bolometric luminosity. We concluded that its cool M-type spectrum was produced in a pseudo-photosphere or optically thick wind formed during a high mass loss episode.

A recent spectrum, twenty years later, reveals another dramatic change. Its spectrum is now that of a much warmer star consistent with the warmer photosphere and colors of 50 years ago. In this paper we discuss its remarkable spectral changes reminiscent of the variability of $\rho$ Cas (Lobel et al. 2003), but on much longer time scales. Variable A was an obvious target for spectroscopy and imaging with the Spitzer Space Telescope. Its resulting spectral energy distribution $(.4 \mu \mathrm{m}$ to $24 \mu \mathrm{m})$ also shows unexpected changes, especially at the long wavelengths, most likely corresponding to the changes in its wind and its impact on its circumstellar medium. In this paper we combine all of the available spectroscopy, multiwavelength photometry and imaging to reconstruct the changes in its wind and circumstellar material over the past twenty years. In the next section we present our multi-wavelength observations. The current spectrum and its light curve and spectral energy distribution are described in $\S 3$ and $\S 4$. In $\S 5$ we discuss Var A's "eruption", its duration, and the changing structure of its wind and circumstellar nebula, and in the last section we review its relationship to other cool hypergiants and the possible origins of their instability.

\section{Observations and Data Reduction}

Our new observations include blue-red groundbased spectra, polarimetry, and spacebased near- and mid-infrared photometry and spectroscopy. 


\subsection{Spectroscopy with the MMT}

Spectra of Variable A were obtained in 2003 and 2004 with the refurbished MMT now with a single 6.5 - meter mirror. The same dual channel spectrograph was used as in 1985 but with a long-slit 1".0 wide. Spectra were observed in both the blue and red channels with different gratings resulting in a range of wavelength coverage and spectral resolution. The journal of observations is in Table 1 . The spectra were all reduced in the standard way in $\mathrm{IRAF}^{1}$ and were flat-fielded, sky subtracted and flux and wavelength calibrated. The S/N of the final spectra is wavelength dependent but is typically $\sim 30$ in the continuum and 80 to 90 for the strong $\mathrm{H} \alpha$ and $\mathrm{H} \beta$ emission lines.

\subsection{Polarimetry}

Polarimetry at visual wavelengths was performed on 2004 September 27 using OptiPol at the Mount Lemmon Observing Facility $1.5 \mathrm{~m}$ telescope. OptiPol is a CCD imaging polarimeter using a Wollaston prism in combination with a rotatable achromatic half-waveplate. Images in perpendicular polarizations are split by the Wollaston prism and placed on opposite halves of the CCD. Two polarization standards, HD 204827 (polarized) and HD 212322 (unpolarized), (Schmidt et al. 1992) were observed. Due to the faintness of Var A in the visual, we were only able to obtain an upper limit of $P \leq 15 \%(3 \sigma)$ in the $\mathrm{R}$ and I filters (combined). Interstellar polarization could be present, but would very likely be much less than our upper limit.

Photometry was extracted from the polarization data by combining the signal at all waveplate positions for each filter. The resulting photometry was placed on the CousinsKron system by observing Landolt standards (Landolt 1983) in the same manner as the polarization standards. The VRI magnitudes are included in Table 2.

\section{3. $\quad$ Spitzer Observations}

Observations of Variable A were made using all four bands of the Infrared Array Camera (IRAC) on 2004 January 9 and were repeated on 2004 July 22, August 16 and 2005 January 1 yielding four epochs of data as part of GTO Program ID 5 to map M33 (P.I Gehrz). The

\footnotetext{
${ }^{1}$ IRAF is written and supported by the IRAF programming group at the National Optical Astronomy Observatories (NOAO) in Tucson, Arizona. NOAO is operated by the Association of Universities for Research in Astronomy (AURA), Inc. under cooperative agreement with the National Science Foundation
} 
IRAC instrument (Fazio et al. 2004) uses four detectors at 3.6, 4.5, 5.8, and 8.0 $\mu \mathrm{m}$. All four detector arrays are 256x256 pixels in size with mean pixel scales of 1.221, 1.213, 1.222, and $1.220 \mathrm{arcsec} / \mathrm{pixel}$ respectively. The M33 observations used a 3 point cycling $\sim 11$ pixel dither for each position, and the integration time was 10.4 secs per frame.

The raw data were processed and flux calibrated with version 11.4 of the Spitzer Science Center (SSC) pipeline. Details of the calibration and raw data processing are specified in the IRAC Pipeline Description Document ${ }^{2}$, version 1.0. Post-BCD(Basic Calibrated Data) processing was carried out using the 2005 May 9 Linux version of the SSC MOPEX ${ }^{3}$ software (Makovoz, Khan \& Moshir 2005). Five steps of MOPEX were implemented: Cosmetic Fix, Final Flat Fielding, Background Matching, Outlier Detection, and Mosaicking. The photometry was measured using a modified version of the ATV IDL photometry package. The field is relatively empty, therefore a source and sky annulus was chosen that corresponds to the apertures used in the pipeline calibration procedures so no aperture correction was necessary. A median sky annulus was used and uncertainties were calculated using the prescription in Reach et al. (2005). The resulting mean magnitudes from the four observations are in Table 2.

Observations with the Infrared Spectrograph (IRS) were made using the short $(5-15 \mu \mathrm{m})$ and long wavelength $(14-28 \mu \mathrm{m})$ low resolution modules on 2004 August 31 with slit widths of $3^{\prime \prime} .7$ and 10!.7, respectively. The observations consisted of 3 cycles of 60 second ramps (effective exposure time) and 3 cycles of 120 second ramps in the short and long wavelength modules respectively. The raw data were processed and flux calibrated with version 12.0 of the SSC pipeline. Details of the calibration and raw data processing are specified in the IRS Pipeline Description Document ${ }^{4}$, version 1.0. Spectra were extracted from the BCD frames using the Spitzer IRS Custom Extractor (SPICE) V1.1B16. Individual spectra were then corrected for bad pixels scaled to the mean, averaged together, and saved in a SPICE-like format. Uncertainties were estimated from the standard deviation (sample variance).

Images of M33 at 24, 70, and $160 \mu \mathrm{m}$ were obtained with the Multiband Imaging Photometer for Spitzer (MIPS) on 2003 December 29, 2005 February 03, and 2005 September 05. A region approximately $1^{\circ} \times 1^{\circ}$ centered on M33 was covered with 4 scan maps. Each map consisted of medium rate scan legs with cross scan offsets of $148^{\prime \prime}$ between each leg. The length of the scan legs varied from 0.75 to 1.0. The raw data were processed and flux calibrated with version 3.02 of the University of Arizona MIPS instrument team Data Analysis

\footnotetext{
${ }^{2}$ ssc.spitzer.caltech.edu/irac/dh/PDD.pdf

${ }^{3}$ Mosaicing and Point Source Extraction

${ }^{4}$ ssc.spitzer.caltech.edu/irs/dh/irsPDDmar30.pdf
} 
Tool (Gordon et al. 2005). Extra processing steps on each image were applied before mosaicking using programs written specifically to improve the reductions in large, well-resolved galaxies. The first epoch $70 \mu \mathrm{m}$ observations (29 Dec 2003) were taken before the operating parameters of this array were finalized, and therefore suffer from significantly larger detector transients than the second epoch data. All three epochs were used for the final mosaics at 24 and $160 \mu \mathrm{m}$, but only the 2nd and 3rd epoch data were used for the final $70 \mu \mathrm{m}$ mosaic. The mean magnitude at $24 \mu \mathrm{m}$ is included in Table 2 ; however, Var A was not detected at either 70 or $160 \mu \mathrm{m}$, so only upper limits are given for those wavelengths.

\section{The Spectrum}

The spectrum of Variable A observed in 1985 and 1986 (HJG) was that of an M-type star with strong $\mathrm{TiO}$ bands and a weak $\mathrm{H} \alpha$ emission line. This spectrum was somewhat of a surprise at that time because the other Hubble-Sandage variables in M31 and M33 were hot stars (Humphreys 1975, 1978) and belonged to the group of unstable massive stars we now call Luminous Blue Variables (see Humphreys and Davidson 1994 for a review). Assuming that Var A's normal state was that of an F-type supergiant, we attributed this M-type spectrum to the formation of a cool optically thick wind corresponding to its rapid decline in apparent brightness and color shift 30 years earlier.

Our recent spectra from 2003 and 2004 reveal another dramatic shift (see Figure 1). The $\mathrm{TiO}$ bands are gone. The spectrum has returned to a much warmer apparent temperature with strong hydrogen emission, absorption lines appropriate for an early $\mathrm{F}$ type to an early G-type star, depending on which lines are used, plus unusual emission lines including K I $(\lambda \lambda 7665,7699)$, the near-infrared Ca II triplet $(\lambda \lambda 8498,8542)$ and [Ca II] at $\lambda 7292$ and $\lambda 7324$ 5 Thus the star has returned to its previous warmer state; although, at this time it is not possible to know if the absorption lines are representative of the star's true photosphere after the dissipation of its cool, dense cirumstellar envelope or are formed in a warm wind which is consistent with the range of identified lines. Var A's recent optical colors (Table 2) are consistent with its much warmer spectrum; however, although it is no longer red, Var A has remained faint.

The strong emission lines and representative absorption lines are listed in Table 3 with their Heliocentric velocities and equivalent widths. Because of the low resolution many of the absorption lines are blended. The expected Heliocentric velocity of Var A at its distance from

\footnotetext{
${ }^{5}$ The Na I 'D' lines may also be in emission, although measurements in that spectral region are complicated by subtraction of the strong night sky lines.
} 
the center of M33 would be about $-130 \mathrm{~km} \mathrm{~s}^{-1}$ from the $\mathrm{H}$ I radial velocity map (Plate 6) in Newton (1980). With respect to this expected velocity, most of the emission and absorption lines are blue shifted which could be due to expansion of the ejecta or to the star's systemic motion, while the K I emission is redshifted. There are also several unidentified emission and absorption lines, and some of the stronger ones are listed in Table 4.

Var A still has an extensive but low-density circumstellar envelope responsible for its strong hydrogen and peculiar emission. Both the $\mathrm{H} \alpha$ and $\mathrm{H} \beta$ emission lines show prominent wings extending to $\pm 800-900 \mathrm{~km} \mathrm{~s}^{-1}$ (see Figure 2) presumably due to Thomson scattering. There is no obvious associated P Cygni absorption; although, at this resolution it may be difficult to tell. Emission lines of K I, the Ca II infrared triplet and [Ca II] are rare in astronomical spectra, but are observed in some of the other "cool hypergiants" with extensive circumstellar material such as IRC+10420 (Ca II, [Ca II], K I; Jones et al 1993, Humphreys et al 2002) and VY CMa (K I; Wallerstein 1958 and numerous subsequent papers). K I emission is only weakly present in the ejecta of IRC +10420 and also in $\rho$ Cas (Lobel 1997). However, in VY CMa it is not only the strongest emission line, but is also stronger there than in any other known late-type star with peak fluxes several times the continuum. In Var A, the $\mathrm{K}$ I lines are second only to $\mathrm{H} \alpha$ and $\mathrm{H} \beta$ in relative strength with peak fluxes about 1.5 times the continuum level. Their doublet ratio also indicates that the lines are optically thick as in VY CMa, and also like VY CMa, the $\lambda 7699$ line is the stronger. Figure 3 shows the region around the $\mathrm{K}$ I lines.

$\mathrm{K} \mathrm{I}$ emission is normally attributed to resonance scattering. However, averaging over directions in a simple model, resonance scattering neither augments nor diminishes the net emergent flux; apparent "emission" must be balanced by an equal amount of "absorption", possibly redshifted or blueshifted or visible along a different line of sight. In a recent paper on VY CMa, Humphreys et al. 2005 (see the Appendix and their Figure 14) suggested a model wherein the K I "emission" component dominates in the emergent spectrum from multiple scatterings from large, dusty condensations in a roughly spherical inhomogeneous shell. In that model, lines of sight where resonance scattering would produce net "absorption" are preferentially hidden because they occur in denser localities, while lines of sight with net "emission" are more favorable for photon escape. (For a more complete description, see the paper cited above.) The K I emission lines in Var A are not as extreme as in VY CMa, but their ratios indicate that they are produced in similar conditions. The redshift of these lines relative to other features (Table 3) may be difficult to explain in this model, but this depends on geometrical details. Alternatively, the gas and dust around Var A may be non-spherical and we may view it from a direction that favors the "emission" component of resonance scattering. As we will see in $\S 5$ a non-spherical geometry is likely. The probable presence of large, dusty condensations will be relevant to our later discussion in sections 4 and 5 . 
Like $\mathrm{K} \mathrm{I}$, [Ca II] is rarely observed in emission because the atoms are normally collisionally de-excited back to the ground state that produces the $\mathrm{Ca}$ II $\mathrm{H}$ and $\mathrm{K}$ lines. The transition that produces the Ca II infrared triplet emission leaves the atoms in the upper level for the forbidden lines, some of which must be radiatively de-excited to produce the forbidden emission. Strong emission from the Ca II infrared triplet and [Ca II] emission are also seen in the spectrum of IRC +10420 . Therefore, in two of our previous papers on IRC +10420 (Jones et al 1993, Humphreys et al 2002), we estimated the relative number of de-excited photons from the ratios of their combined equivalent widths ([Ca II] to $\mathrm{Ca} \mathrm{II}^{6}$ ) to their corresponding continuum fluxes. Following this same procedure for Var A, we find that $\approx 27 \%$ of the photons produce the [Ca II], and $n_{e}$ is only 2.7 times the critical density for radiative de-excitation. This is very similar to our results for IRC +10420 , and shows that both stars possess extended regions of very low density gas beyond their dense winds or photospheres.

The IRS spectra from 5 to $15 \mu \mathrm{m}$ and 14 to $28 \mu \mathrm{m}$ show that Var A also has prominent silicate emission at $9.7 \mu \mathrm{m}$ and $18 \mu \mathrm{m}$. The $9.7 \mu \mathrm{m}$ feature is quite strong in emission, at least as strong as the $9.7 \mu \mathrm{m}$ emission feature in the Galactic hypergiant IRC +10420 (Jones et al. 1993), see Figures 5 and 6 . The ratio of the 9.7 to $18 \mu \mathrm{m}$ emission features is typical for optically thin dust shells in Galactic red giants and supergiants (e.g. Little-Marenin \& Little 1990).

\section{The Light Curve and Spectral Energy Distribution}

Figure 4 shows Var A's light curve from 1950 to the present based on the work by Hubble and Sandage (1953) and Rosino and Bianchini (1973) together with the photoelectic and CCD blue and visual photometry between 1977 and 1986 from HJG, photographic B photometry from 1982 to 1990 (Kurtev et al. 1999), and CCD photometry from 2000-01 (Massey 2005). The older photographic color index has been converted to an approximate $B-V$ color (Allen 1963). The most recent data for 2004 are derived from integration of our flux calibrated spectra over the standard band passes for B, V, and R magnitudes, and from the VRI frames used for the polarimetry observations. These two independent methods give consistent results. Hubble and Sandage (1953) originally suggested that the decline of $\sim 3$ to 3.5 magnitudes in the photographic blue was consistent with a shift in bolometric correction and the corresponding change in color index. The 1985-86 spectrum confirmed that the star's

\footnotetext{
${ }^{6}$ The Ca II $\lambda 8662$ line was not covered by our spectra. Therefore, we estimated its equivalent width from the expected line ratios of the Ca II triplet compared with the measurements of the other two lines.
} 
apparent energy distribution had indeed shifted to a much cooler temperature. However, between 1954 and 1986 the star faded an additional two magnitudes in the blue which we attribute to extinction by circumstellar dust.

In Table 2 we summarize the multi-wavelength photometry for Var A from HJG, an unpublished JHK measurement from 1992 by Gehrz and C. Woodward, optical photometry from Massey(2005) and more recent photometry from the calibrated optical spectrum and polarimetry, JHK photometry from the 2MASS All-Sky Point Source Catalog, and the mean 3.6 to $8.5 \mu \mathrm{m}$ IRAC measurements from Spitzer. The current $24 \mu \mathrm{m}$ point is from the Spitzer MIPS observation. Figure 5 shows Var A's present broadband spectral energy distribution together with the IRS spectrum compared with its energy distribution from 1986. This comparison reveals several very significant changes.

The most oustanding difference is the large drop in flux by a factor of three at $10 \mu \mathrm{m}$, and by $\sim 5$ times at $18 \mu \mathrm{m}$. Note that in 1986 our measured $10 \mu \mathrm{m}$ flux showed that the star's total energy output had remained constant and that it was still as luminous as at its maximum light in 1950. The recent decline in the long wavelength flux has important implications for possible dissipation and/or destruction of the reradiating dust and for any model for the star's changing wind and distribution of the circumstellar material. Consequently we have carefully checked the earlier mid-infrared photometry and the IRAS data used by HJG, and are confident in the earlier, higher fluxes for the following reasons.

There is clearly a source in the IRAS $12 \mu \mathrm{m}$ coadded maps at the position of Var A. A subsequent analysis of IRAS observations of M33 by Rice et al. (1990) reached a similar conclusion. Rice et al. derived a $12 \mu \mathrm{m}$ flux (.10 Jy) and an upper limit for the $25 \mu \mathrm{m}$ flux (.09 Jy) consistent with the values we reported in HJG. There is also no evidence in the current Spitzer observations of another source, such as a bright HII region, that could have contributed more than $25 \%$ to the IRAS fluxes.

The ground based $10 \mu \mathrm{m}$ magnitude used in HJG (see Table 2 this paper) was derived from data from four separate observing runs. These observing runs on the IRTF and WIRO telescopes resulted in one $4 \sigma$ detection and three $3 \sigma$ upper limits. Combining these observations, HJG computed a $4 \sigma$ value for the $10 \mu \mathrm{m}$ flux of Var A which is completely independent of, but entirely consistent with, the IRAS $12 \mu \mathrm{m}$ flux.

Finally, we note that the luminosity of Var A in the mid-infrared reported in HJG was very close to the pre-outburst optical luminosity of the star. Observations of yellow and red hypergiants as well as the Luminous Blue Variables show that these stars maintain essentially constant luminosity as they undergo variations in their apparent temperature (see Humphreys and Davidson 1994). If dust formed in the material shed by Var A and completely 
covered the star, then it would absorb all of the stellar luminosity and reradiate it in the infrared, as observed by HJG. The present decrease in the $10 \mu \mathrm{m}$ and $18 \mu \mathrm{m}$ flux compared with Var A's luminosity at maximum light means that a significant fraction of the star's light must be escaping elsewhere. The lack of measurable flux at even longer wavelengths (70 and $160 \mu \mathrm{m})$ rules out the presence of cooler dust that could be re-radiating the missing flux. Furthermore there has not been sufficient time for the circumstellar material to have expanded to those much larger distances from the star.

The fading of its near-IR flux (from 2MASS) very likely corresponds to a combination of the shift in the energy distribution and the possible dissipation of warm dust nearer the star due to changes in the wind as its density decreased and radiation from warmer layers escaped. The optical colors between 2000 and 2004 show the object getting slightly bluer which may be due to a continued decrease in wind density. The recent photometry also shows that the return to a warmer apparent photosphere was not accompanied by a visual brightening. The most likely explanation is that its present faintness in the optical is due to obscuration by circumstellar dust, although it is not red, suggesting that the circumstellar extinction is relatively neutral due to grains larger than is typical for interstellar and circumstellar dust, but still consistent with the silicate emission. Compared with its visual maximum, when Var A apparently had a comparable temperature, this implies that it now has approximately four magnitudes of circumstellar extinction in the visual.

Variable A's eruption, the changes in its wind and circumstellar material are discussed in the next section.

\section{Discussion - The Wind and Circumstellar Material in Transition}

Our current observations reveal some remarkable changes in the spectrum and energy distribution of Variable A. Although, we do not have a more complete spectroscopic and

photometric record over the past 20 years, our observations do allow us to put together a picture of a very luminous, unstable star experiencing major changes in the structure of its wind and circumstellar material at the end of a high mass loss event.

Variable A still had its cool, dense wind and was in a high mass loss phase 35 years after it rapid decline in 1951. Thus it was in "eruption" for at least that long. The photometric record also shows that Var A faded two magnitudes between 1954 and 1986 presumably due to the formation of dust, and may have created an additional two magnitudes of circumstellar extinction since then. Our recent spectrum 20 years later shows that the star's F-type photosphere has returned. When did this transition occur? The star has remained faint 
even though the spectrum has presumably recovered. The variation in its near-infrared photometry may correspond to changes in the wind accompanying the subsidence of its cool, dense wind phase. The 2MASS observations obtained in Dec. 1997 show that Var A had faded significantly in the near-infrared. However, the JHK photometry from 1992 does not show any change from the earlier data in 1986. Assuming that the near-infrared variation is due to the wind in transition, then Var A's eruption or dense wind stage may have lasted between 41 and 46 years!

How long the transition back to a warmer temperature may have taken is uncertain, but we note that the initial formation of the dense wind took approximately a year based on its 1950's light curve or at most two years if we include the brief recovery and second decline. Using the record of $\rho$ Cas, which shows similar outbursts or shell episodes as an example, the onset and recovery timescales are comparable, and in $\rho$ Cas they occur very rapidly, in only a couple of months. Thus many of the changes we discuss below may have occurred over only one to two years at most for the spectroscopic changes and perhaps up to five years (1992 to 1997) or so for the onset of the changes in the distribution and structure of the circumstellar material. The transition in the wind from an M-type false-photosphere to a warmer F-type star, could reasonably have occurred on even shorter timescales. Although we do not have a direct measurement of Var A's wind speed, the expansion velocities for the winds and ejecta in IRC+10420 and VY CMa are $40-60 \mathrm{~km} \mathrm{~s}^{-1}$ and $35 \mathrm{~km} \mathrm{~s}^{-1}$ for the envelope expansion during $\rho$ Cas's recent episiode. Assuming $50 \mathrm{~km} \mathrm{~s}^{-1}$ for the wind speed, Var A's envelope could have made these transitions in as short as 3/4's of a year, consistent with its variations during the early 1950's.

To explain Var A's observed energy distribution in 1986, HJG proposed a simple model of a cool, dense false-photosphere with an obscuring torus and reflection nebulae at the poles. This model combined extinction by circumstellar dust with the blueing effect of scattering by dust grains and assumed that most of the flux was radiated in the mid-infrared. Most of the visible light was scattered and not viewed through the intervening material. In this model the visible light would very probably have been more highly polarized than the upper limit of $15 \%$ reported here. There was no optical polarimetry in 1986, so we cannot check this model or verify that there has been a change.

Despite its warmer apparent temperature and the corresponding change in the bluevisual energy distribution, Var A has not brightened in the visual, and comparison with its maximum light implies $\approx 4$ magnitudes of circumstellar extinction currently in the line of sight. HJG showed that the visual interstellar extinction for Var A was about 0.6 to 0.8 mag which is typical for stars in M33. With its current $B-V$ color of 0.8 to 0.9 this suggests that its current true color is 0.6 to 0.4 , appropriate for a late F-type star, and in comparison with 
its colors at maximum light $(\sim .4)$, implies virtually no circumstellar reddening in the visual. As we mentioned previously, the dust grains must be large enough to provide the neutral extinction in the visual which is also consistent with the scattering requirements for the $K$ I emission. Our polarimetry upper limit of $15 \%$ suggests that the optical light at present is not due mostly to scattered light. If we adopt $30 \%$ for the polarization of a star completely blocked by circumstellar dust and visible only in reflected light (Johnson and Jones 1991), then at most half the visible light from Var A is from scattered photospheric radiation. The polarimetry also rules out any current asymmetries such as bipolar lobes although reflection nebulosity in a more spherical distribution could still be present.

The most dramatic change in Var A's energy distribution is the apparent decrease in its total flux. Since it is highly unlikely that the total luminosity of the star would have declined by more than a magnitude, the energy must now be escaping in some direction other than along our line of sight. Several massive stars are now known to have asymmetrical winds or bipolar outflows. The most notable is $\eta$ Car with a latitude dependent wind (Smith et al 2002) that is both faster and denser at the poles, although $\eta$ Car is a very different kind of star. However, given the evidence from other evolved, massive stars including the cool hypergiants like IRC+10420 and VY CMa, for irregularities and density variations in their circumstellar ejecta, it is likely that Var A's dusty shroud is not uniform or completely opaque in all directions. In the remaining discussion of Var A's wind and circumstellar material we will assume that the missing radiation is escaping through large, low density regions, even holes, in the obscuring material, and based on the timescales discussed above for the duration of the eruption and the recovery, we'll assume that Var A has been in this warmer state for approximately ten years.

HJG fit Var A's 1986 mid-infrared flux by a $370^{\circ} \mathrm{BB}$ which implies a dusty zone or shell with a radius of $\sim 400 \mathrm{AU}$ from the star. The energy distribution had a significant near-infrared flux due to warmer dust closer to the star, so the dusty zone probably extended from 100 to 400 AU or so. Figure 4 shows that Var A slowly faded over 30 years due to increased circumstellar obscuration presumably as the amount and density of the obscuring material increased. HJG had estimated a mass loss rate of $2 \times 10^{-4} \mathrm{M}_{\odot} \mathrm{yr}^{-1}$ from Elitzur's (1981) formulation assuming radiation coupling to the grains. But when Var A's optically thick wind ceased and quickly subsided back to a warmer state, then its mass loss rate would very likely have decreased as well. For example, the mass loss rates of the LBV's during their eruptions are typically 10 to 100 times that during their quiescent stage, and the normal mass loss rates of $\rho$ Cas and HR 8752, two hypergiants of comparable luminosity and temperature, are $\sim 10^{-5} \mathrm{M}_{\odot} \mathrm{yr}^{-1}$. In addition to a less dense wind and a lower mass loss rate, Var A may also have a higher wind speed now. Wind speeds of 100 to $200 \mathrm{~km}$ $\mathrm{s}^{-1}$ are typical of normal A to F-type supergiants. Following HJG's procedure, using Var 
A's apparent luminosity inferred from its current $10 \mu \mathrm{m}$ flux, and assuming that the optical depth is near one in our line of sight, we find a current mass loss rate of $\sim 6.7 \times 10^{-5} \mathrm{M}_{\odot}$ $\mathrm{yr}^{-1}$ for a $50 \mathrm{~km} \mathrm{~s}^{-1}$ wind. But if the wind speed is higher, the mass loss rate will be $\sim 2-$ $3 \times 10^{-5} \mathrm{M}_{\odot} \mathrm{yr}^{-1}$.

In the approximately 10 years since its transition, Var A's lower density and possibly faster wind would have reached 100 to $300 \mathrm{AU}$, the region of the dusty zone. Consequently, the dusty material may not be replenished as efficiently as in the previous dense wind, higher mass loss stage. So as the dusty zone has continued to expand during this same period, the lower density regions or gaps will also have enlarged allowing more radiation to escape. If the dusty distribution is flattened, as HJG suggested, this is most likely to occur at the poles.

Therefore we propose that large dusty condensations in a flattened distribution, possibly a torus, currently obscure our direct view of Var A. The K I emission lines are produced by resonance scattering in this dusty zone. In addition, a very low density gas or wind responsible for the hydrogen, Ca II, and peculiar [Ca II] emission fills the region between the star's photosphere and the dusty zone. More than half of the radiation is escaping from our line of sight, presumably from low density regions, all or most of which must be out of our line of sight. Furthermore, the polarimetry measurements rule out a strongly bipolar structure with asymmetrically scattered light. This then suggests a unique geometry even if the gaps are restricted to the polar regions. The dusty zone must either be nearly aligned with our line of sight in such a way to also block most of the escaping radiation from being reflected back into our line of sight or else there is very little reflecting nebulosity.

\section{Discussion - Variable A and the Cool Hypergiants}

HJG derived a total luminosity for Var A of $5 \times 10^{5} \mathrm{~L}_{\odot}$ based on both its luminosity at its visual maximum $\left(\mathrm{M}_{v}=-9.4\right.$ to $\left.-9.6 \mathrm{mag}\right)$ and its mid-infrared flux in 1986. As we've already emphasized the two agreed. Its position on the HR diagram in its two states, cool dense wind and warm photosphere or wind, can be seen in Figure 4 in HJG and also on Figure 14 in Humphreys et al (2002) together with other very luminous evolved cool hypergiants some of which have already been mentioned in this paper. Var A is one of several luminous stars in our galaxy and others that define the upper luminosity boundary for evolved cool stars (Humphreys and Davidson 1979, 1994).

Var A shows spectral and photometric characteristics in common with several of these

stars, especially IRC +10420 and $\rho$ Cas. Spectroscopically, it is most like the post-red supergiant IRC +10420 with its strong hydrogen emission, and the Ca II and [Ca II] emission 
lines. IRC+10420 has a large infrared excess, is a powerful OH maser and has an extended and complex reflection nebula (Humphreys et al 1997). but it does not have significant circumstellar reddening. Figure 6 shows a comparison of their spectral energy distributions. Var A and IRC+10420 have comparable luminosities with strong $9.7 \mu \mathrm{m}$ silicate emission features and gently rising continua from $1-8 \mu \mathrm{m}$. IRC +10420 has high interstellar extinction in the visual that causes the rapid drop in flux at wavelengths shorter than $1 \mu \mathrm{m}$. Emission from warm dust near the star contributes to its excess radiation longwards of $2 \mu \mathrm{m}$. This is observed in other evolved stars with extensive circumstellar ejecta, and may also be the case for Var A (see HJG). The $18 \mu \mathrm{m}$ silicate feature is unusually strong in IRC+10420 compared to the more "normal" feature in Var A. Based on laboratory investigations, Nuth and Hecht (1990) suggest that a relatively stronger $18 \mu \mathrm{m}$ feature indicates grains that are more highly processed, implying that the dust associated with Var A formed relatively recently and is not the remnant of an old mass-loss phase in the star's evolution. We do not have any record of a prior cool, dense wind state in IRC+10420, although Humphreys et al (2002) concluded that with its very high mass loss rate $\left(3-6 \times 10^{-4} M_{\odot} \mathrm{yr}^{-1}\right), \mathrm{IRC}+10420$ 's current warm wind $(\approx$ $8000-9000^{\circ}$ ) is optically thick. Interestingly, the light curves of both Var A and IRC+10420 show a long period during which they slowly increased in apparent brightness (see Hubble and Sandage 1953, Gottleib and Liller 1978). In Var A this slow rise in brightness culminated in its 1950's ejection episode, while in IRC+10420, the star has been at essentially constant visual brightness since 1970. It is interesting to speculate, that both of these stars were recovering from a previous eruption during which they had suffered significant circumstellar extinction which had slowly dissipated in the preceding decades.

The other example, $\rho$ Cas, is best known for its historical and recent high mass loss episodes during which it produces a cool dense wind with the corresponding spectral changes from a warm F-type photosphere to an M-type with $\mathrm{TiO}$ bands. However, the durations of its episodes are quite short. The most recent in 2000 lasted only 200 days (Lobel et al 2003) while the previous recorded 1945-6 event lasted 1 to 2 years (Beardsley 1953, Bidelman and McKellar 1957). However, $\rho$ Cas and a similar star HR 8752, have very little if any circumstellar dust and neither has any associated visible nebulosity in HST/WFPC2 images (Schuster, Humphreys and Marengo 2005). The much longer duration of Var A's optically thick wind and high mass loss stage may account for its obscuring circumstellar material, although this brings up issues concerning the sustainability of the ejection and the origin of the underlying instability.

de Jager (1998) has suggested that the intermediate temperature hypergiants are postred supergiants which in their evolution to warmer temperatures enter a temperature range $(6000-9000 \mathrm{~K})$ with increased dynamical instability where high mass loss episodes occur. Due to increased mass loss in the red supergiant stage the most massive, most luminous of 
these stars, near the upper luminosity boundary, may lose enough mass to bring them close to the classical Eddington limit $\left((L / M)_{E d d}=4 \pi c G / \kappa\right)$. Then in their post-red supergiant evolution, as the apparent temperature increases above $6500 \mathrm{~K}$, the ionization and opacity increase rapidly, and the "modified Eddington limit" 7 becomes important (see Humphreys and Davidson 1994, Humphreys et al. 2002). When combined with other atmospheric effects such as ionization of hydrogen and pulsation, this leads to high mass loss episodes and very strong winds. Indeed, proximity to the modified Eddington limit may amplify other instabilities making their effects more extreme. IRC +10420, $\rho$ Cas and HR8752 are most often cited as examples of evolved stars in this stage, and Var A undoubtedly belongs to this group.

$\rho$ Cas's recent high mass loss episode was preceded by a period of pulsational instability and Lobel et al (2003) demonstrated that the outburst was initated by the release of ionization energy due the recombination of hydrogen as the atmosphere cooled during the expansion. This mechanism explains the short episodes in $\rho$ Cas, but is it adequate to maintain the long term optically thick wind observed in Var A? Perhaps a deeper instability, triggered by an atmospheric phonomenon, is responsible for maintaining the "eruption" for more than 35 years.

Variable A's high mass loss stage has apparently ended. If in 1950 we had our current ground and spacebased optical and infrared instruments, we could have observed the changing characteristics of its ejecta, the formation and dissipation of dust, over a 50 year period. Nevertheless, despite its present apparent faintness, continued observation of Variable A in the optical and near-infrared is important to monitor further changes. It is the first object of this type for which we have found significant changes in its energy distribution corresponding to a recent high mass loss event. Variable A thus presents us with the opportunity to continue to observe the changing structure of its circumstellar ejecta as it recovers from a long term "eruption".

It is a pleasure to thank Kris Davidson for useful discussions about winds and instabilities in evolved massive stars. We are especially grateful to Phil Massey for providing the UBVRI photometry of Var A in advance of publication from the Local Group Galaxies Survey (Massey et al. 2002). This work was supported in part by the University of Minnesota. It is based on observations made with the Spitzer Space Telescope, which is operated by the Jet Propulsion Laboratory, California Institute of Technology, under NASA contract 1407. Support for the authors was provided by NASA through contracts 1256406 and 1215746

\footnotetext{
${ }^{7} \kappa$ is temperature dependent.
} 
issued by JPL/Caltech.

\section{REFERENCES}

Allen, C. W. 1963, Astrophysical Quantities

Beardsley, W.A. 1961, ApJS, 5, 381

Bidelman, W.P. \& McKellar, A. 1957, PASP, 69, 31

Cohen, M., Witteborn, F. C., Carbon, D. F., Davies, J. K., Wooden, D. H., \& Bregman, J, D. 1996, AJ, 112, 227

de Jager, C. 1998, A\&A Rev., 8, 145

Elitzur, M. 1981, in Physical Processes in Red Giants, ed. I. Iben \& A. Renzini (Reidel, Dordrecht), p. 363

Gordon, K. et al.2005, PASP, 117, 503

Gottleib, E. W. \& Liller, W. 1978, ApJ, 225, 488

Hubble, E. \& Sandage, A. 1953, ApJ, 118, 353

Humphreys, R. M. 1975, ApJ, 200, 426

Humphreys, R. M. 1978, ApJ, 219, 445

Humphreys, R.M. \& Davidson, K. 1979, ApJ, 232, 409

Humphreys, R.M., Jones, T. J. \& Gehrz, R.D. (HJG) 1987, AJ, 94, 315

Humphreys, R.M. \& Davidson, K. 1994, PASP, 106, 1025

Humphreys, R.M., Smith, N., Davidson, K. et al. 1997, AJ, 114, 2778

Humphreys, R.M., Davidson, K., \& Smith, N. 2002, AJ, 124, 1026

Humphreys, R.M., Davidson, K., Ruch, G., \& Wallerstein, G. 2005, AJ, 129, 492

Johnson, J. J., \& Jones, T. J. 1991, AJ, 101, 1735

Jones, T. J., Humphreys, R. M., Gehrz, R. D. et al. 1993, ApJ, 411, 323

Kurtev, R. G., Corral, L. G. \& Georgiev, L. 1999, A\&A, 796 
Landolt, A. U. 1983, AJ, 88, 439

Little-Marenin, I. R., \& Little, S. J. 1990, AJ, 99, 1173

Lobel, A. 1997, Ph.D Thesis, Vrije Univ.

Lobel, A., Dupree, A.K., Stefanik, R.P. et al. 2003, ApJ, 583, 923

Makovoz, D., Khan, I., \& Moshir, M. 2005, PASP, 117, 274

Massey, P., Hodge, P. W., Holmes, S., Jacoby, J., King, N. L., Olsen, K., Smith, C., \& Saha, A. 2002, BAAS, 34, 1272

Massey, P. 2005, private communication

Molster, F. J., Waters, L. B. F. M., Tielens, A. G. G. M., \& Barlow, M. J. 2002, A\&A, 382, 184

Newton, K. 1980, MNRAS, 190, 689

Nuth, J. A., \& Hecht, J. H. 1990, Ap\&SS, 163, 79

Reach et al.2005, PASP, 117, 978

Rice, W., Boulanger, F., Viallefond, F., Soifer, B. T. \& Freedman, W. L. 1990, ApJ, 358, 418

Schmidt, G. D., Elston, R., \& Lupie, O. L. 1992, AJ, 104, 1563

Schuster, M, Humphreys, R. M. \& Marengo, M. 2006, AJ, in press

Smith, N., Davidson, K., Gull, T.R., Ishibashi, K. \& Hillier, D. J. 2003, ApJ, 586, 432

Wallerstein, G. 1958, PASP, 70, 479 


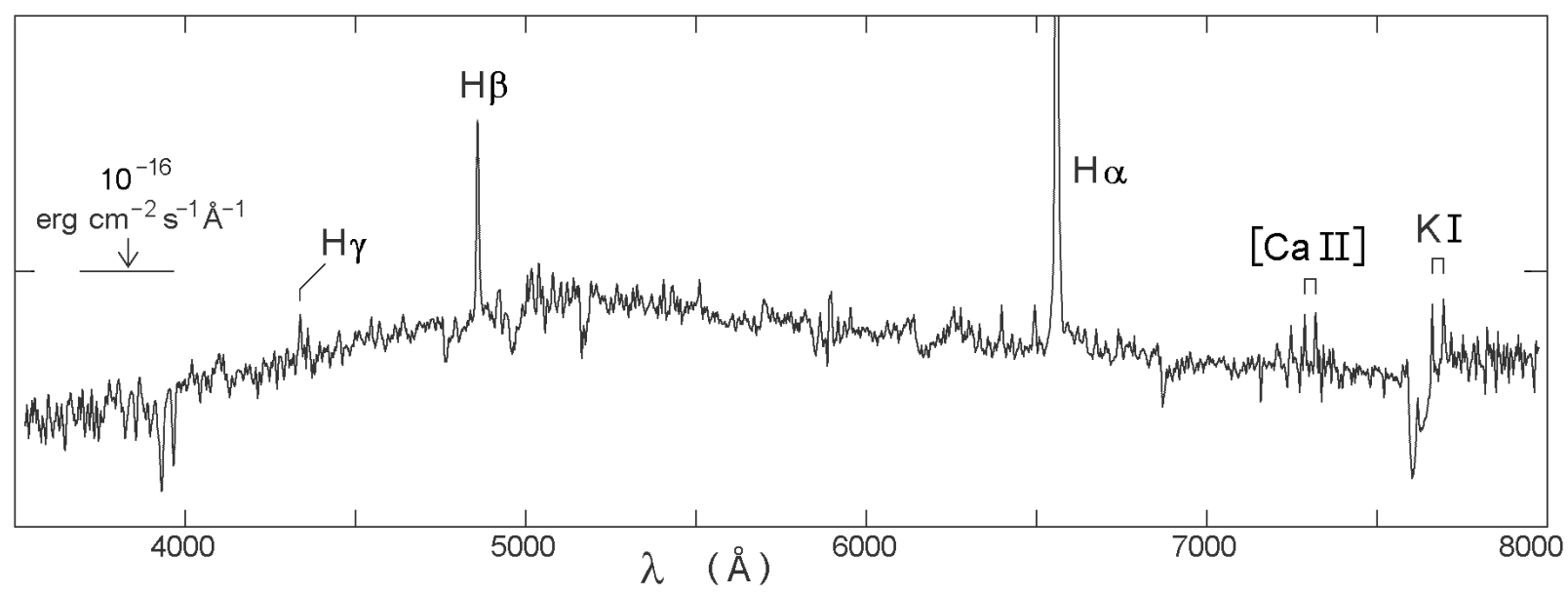

Fig. 1. - The optical spectrum from $\sim 3600 \AA$ to $8000 \AA$. The strongest emission lines are marked. The lower boundary represents zero flux, and a level of $10^{-16} \mathrm{erg} \mathrm{cm}^{-2} \mathrm{~s}^{-1} \AA^{-1}$ is marked near the left side. 


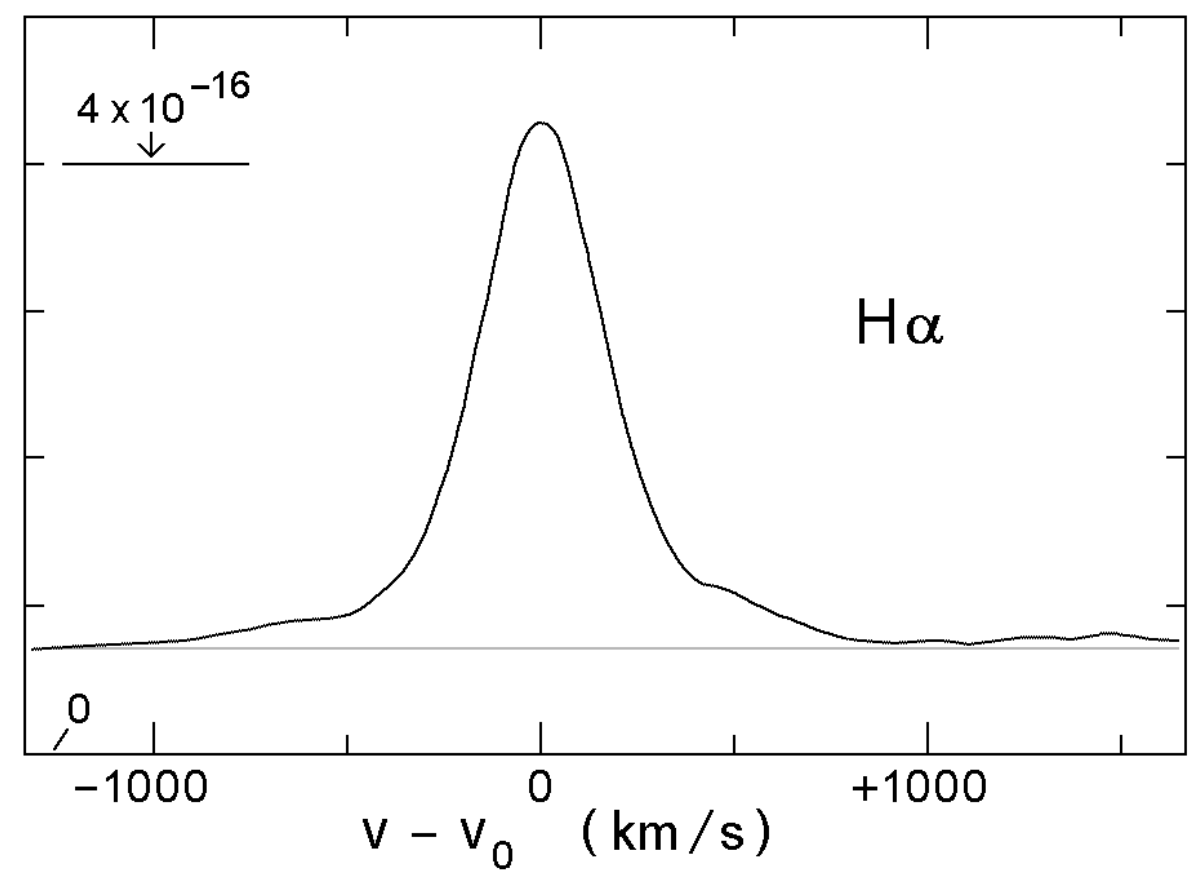

Fig. 2.- The $\mathrm{H} \alpha$ profile showing the broad Thomson scattering wings. The $\mathrm{x}$-axis is relative to the centroid of the profile. $\mathrm{H} \alpha$ has a heliocentric velocity of $-150 \mathrm{~km} \mathrm{~s}^{-1}$. The lower boundary represents zero flux, and a level of $4 \times 10^{-16} \mathrm{erg} \mathrm{cm}^{-2} \mathrm{~s}^{-1} \AA^{-1}$ is marked in the upper left corner. 


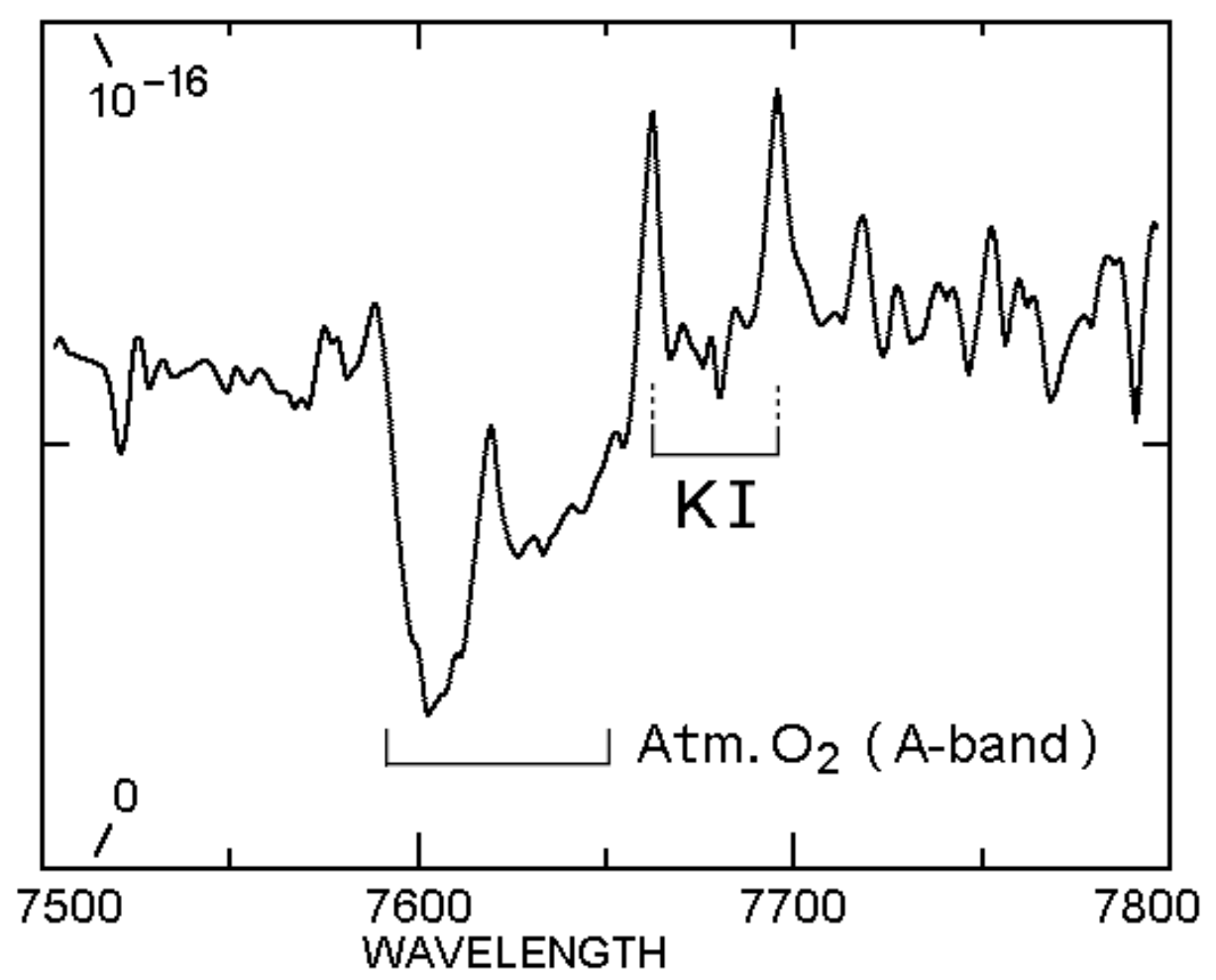

Fig. 3.- The far-red spectrum showing the region of the K I emission lines and the atmospheric A-band. The bottom and top boundaries represent flux levels of zero and $10^{-16} \mathrm{erg}$ $\mathrm{cm}^{-2} \mathrm{~s}^{-1} \AA^{-1}$ respectively. 

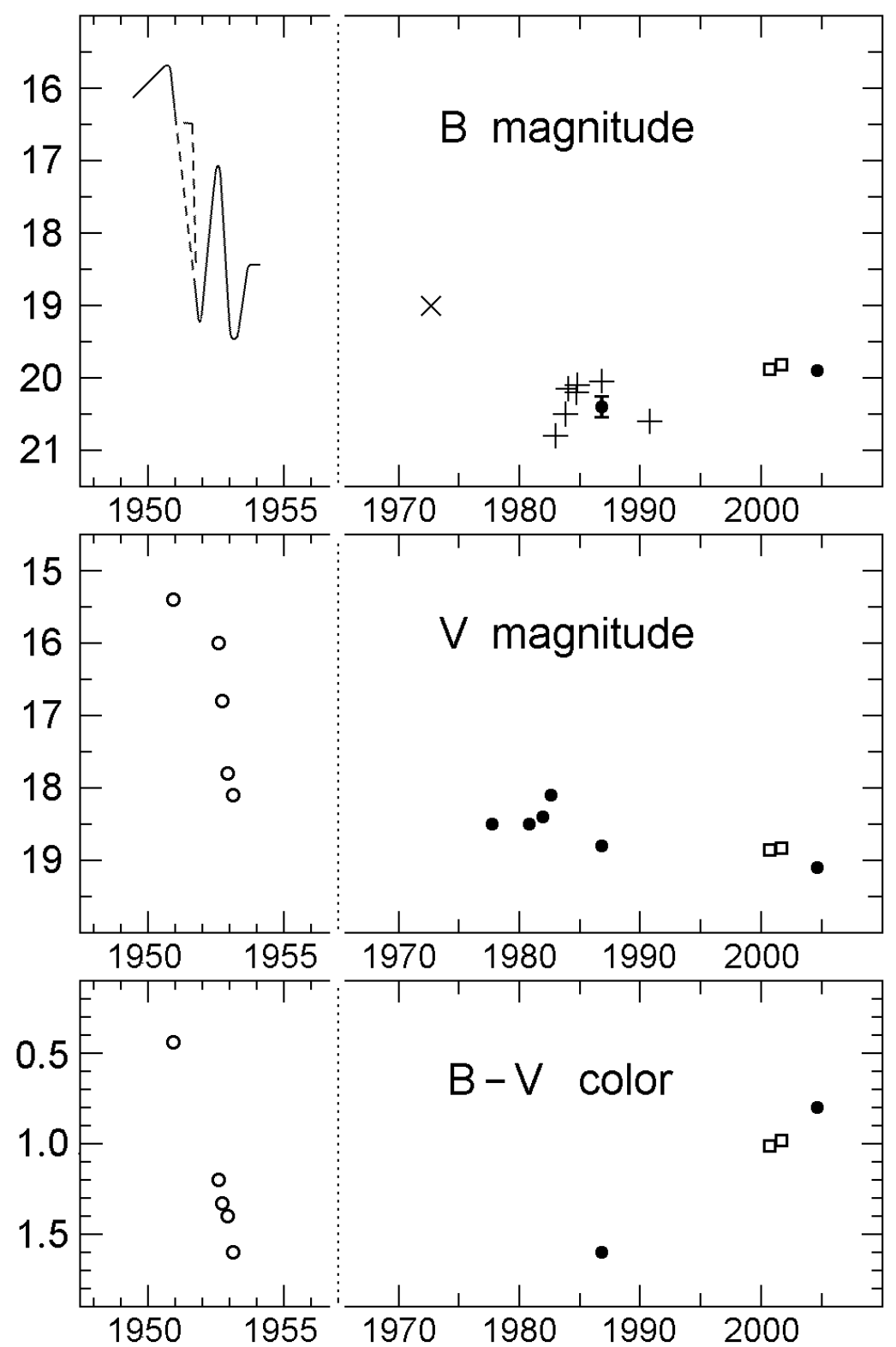

Fig. 4.- Variable A's light curve from 1950 to the present. The upper panel shows the photographic and B band magnitudes. The solid and dashed lines are from Hubble \& Sandage (1953) and Rosino \& Bianchini (1973). The middle and lower panels show the variability in the $\mathrm{V}$ band and the $B-V$ color. The different symbols are from Hubble \& Sandage (1953) open circles, Rosino \& Bianchini (1973) tipped crosses, Kurtev et al. (1999) crosses, Massey (2005) squares, and filled circles from HJG and this paper. 


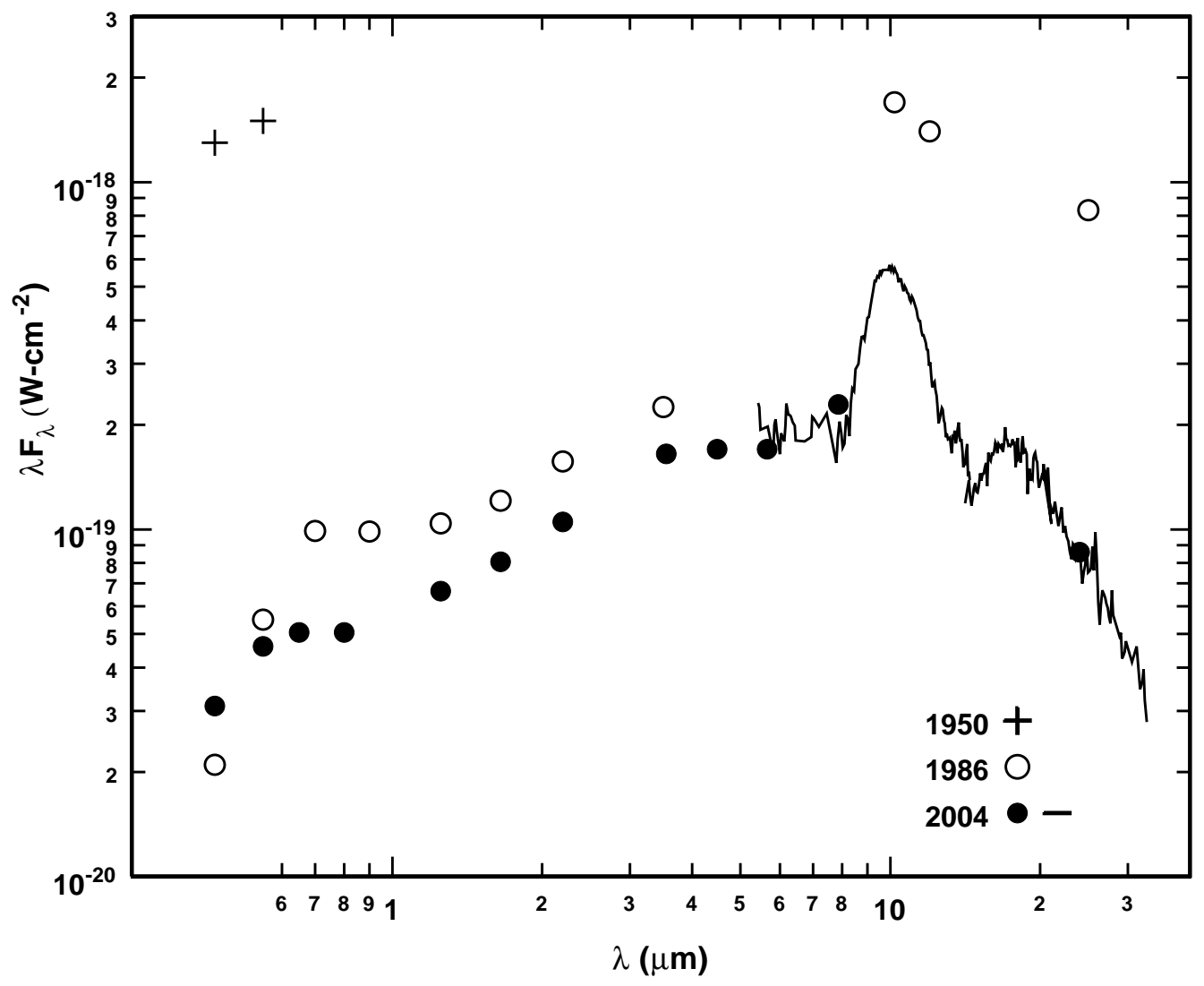

Fig. 5.- Variable A's spectral energy distribution from 1986(HJG) and its current energy distribution from data in this paper. The IRS spectrum from 5 to $28 \mu \mathrm{m}$ is also shown. The crosses show its apparent magnitudes at maximum light from Hubble \& Sandage(1953) transformed to standard B and V magnitudes on the Johnson system. 


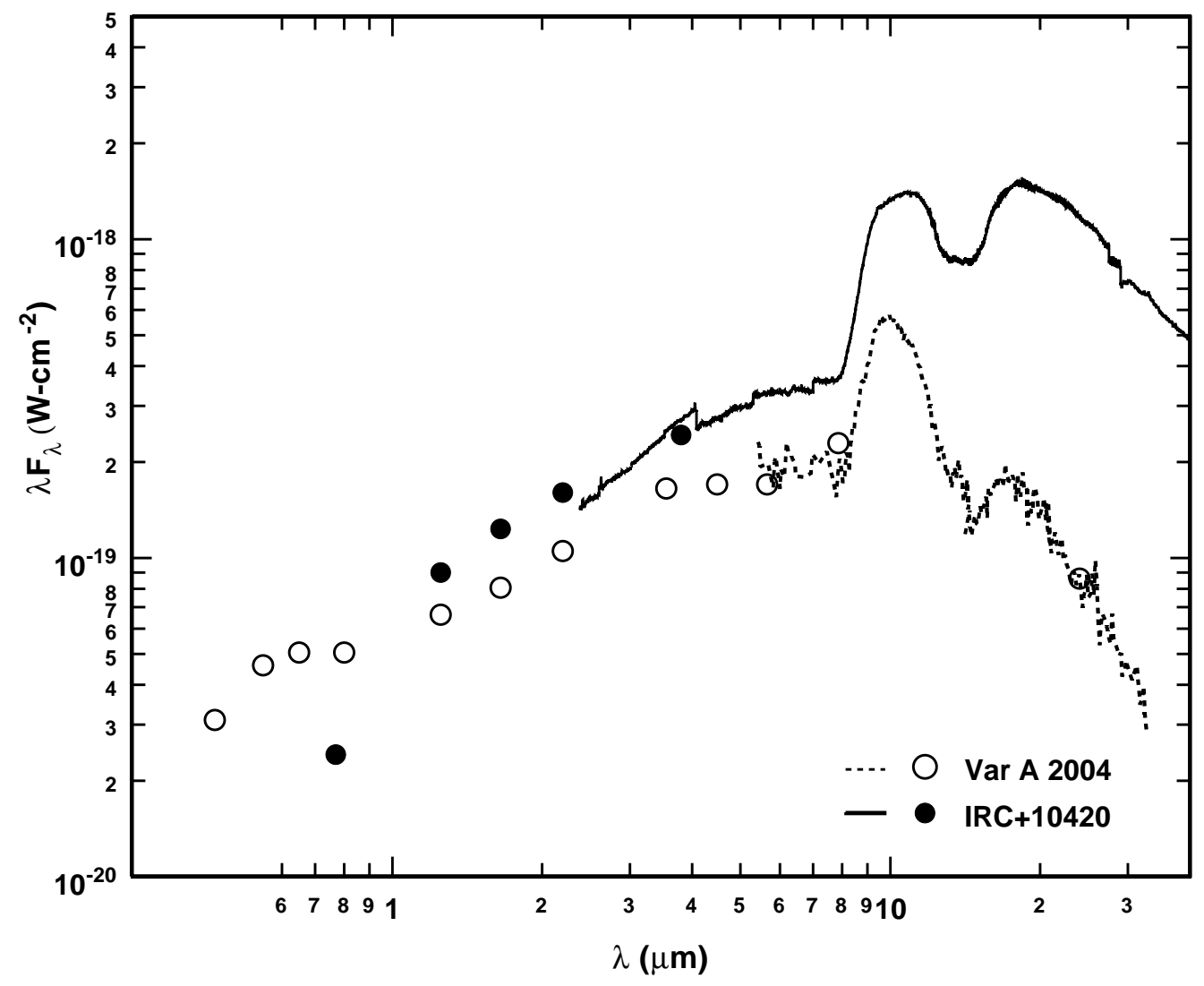

Fig. 6.- A comparison of the spectral energy distributions of Variable A and IRC +10420 in our galaxy shifted to the distance of M33. The spectrum of IRC +10420 from 2 to 30 $\mu \mathrm{m}$ is from ISO data (Molster et al.2002). The rapid drop at the shorter wavelengths in IRC +10420 is due to high interstellar extinction. 
Table 1. Journal of New Observations

\begin{tabular}{|c|c|c|c|c|}
\hline Date(U.T.) & Grating/Filter & $\lambda_{0}$ & Spectral Resolution & Total Integration \\
\hline \multicolumn{5}{|c|}{ a. MMT Double Spectrograph } \\
\hline Nov. 18, 2003 & 6001(red) & $6400 \AA$ & $4.7 \AA$ & $40^{m}$ \\
\hline Nov. 18, 2003 & 2701(red) & $7000 \AA$ & $10.8 \AA$ & $60^{m}$ \\
\hline Nov. 19, 2003 & 300l(blue) & $6000 \AA$ & $6.2 \AA$ & $45^{m}$ \\
\hline Sep. 23, 2004 & 3001(blue) & $6000 \AA$ & $6.2 \AA$ & $80^{m}$ \\
\hline \multicolumn{5}{|l|}{ b. Polarimetry } \\
\hline Sep. 27, 2004 & VRI & - & - & $100^{m}, 80^{m}, 80^{m}$ \\
\hline \multicolumn{5}{|c|}{ c. Spitzer - IRAC } \\
\hline Jan. 9, 2004 & $3.6,4.5,5.8,8.0 \mu \mathrm{m}$ & - & - & $31.2^{s}$ ea ch \\
\hline Jul. 22, 2004 & $"$ & - & - & $"$ \\
\hline Aug. 16, 2004 & $"$ & - & - & $"$ \\
\hline Jan. 1, 2005 & $"$ & - & - & $"$ \\
\hline \multicolumn{5}{|l|}{ d. Spitzer - IRS } \\
\hline \multirow{2}{*}{$\begin{array}{l}\text { Aug. 31, } 2004 \\
,\end{array}$} & $5-15 \mu \mathrm{m}$ & - & - & $366^{s}$ \\
\hline & $14-28 \mu \mathrm{m}$ & - & - & $731^{s}$ \\
\hline \multicolumn{5}{|c|}{ e. Spitzer - MIPS } \\
\hline Dec. 29,2003 & $24,70,160 \mu \mathrm{m}$ & - & - & $238^{s}, 43^{s}, 19^{s}$ \\
\hline Feb. 3, 2005 & $"$ & - & - & $"$ \\
\hline Sept. 5, 2005 & $"$ & - & - & $"$ \\
\hline
\end{tabular}


Table 2. Summary of Photometric Data

\begin{tabular}{|c|c|c|c|c|c|}
\hline Bandpass & $\begin{array}{c}1986 \text { (HJG) } \\
\text { mag. }\end{array}$ & $\begin{array}{l}1992 \\
\text { mag. }\end{array}$ & $\begin{array}{l}1997^{\mathrm{a}} \\
\text { mag. }\end{array}$ & $\begin{array}{l}2000-01^{\mathrm{b}} \\
\text { mag. }\end{array}$ & $\begin{array}{c}2004-05 \\
\text { mag. }\end{array}$ \\
\hline $\mathrm{U}$ & - & - & - & $20.2,20.1 \pm 0.02$ & - \\
\hline B & $20.4 \pm 0.15$ & - & - & $19.9,19.8 \pm 0.01$ & $19.9^{\mathrm{c}}$ \\
\hline $\mathrm{V}$ & $18.8 \pm 0.10$ & - & - & $18.8,18.8 \pm 0.01$ & $19.1^{\mathrm{c}}, 19.1 \pm 0.17^{\mathrm{d}}$ \\
\hline $\mathrm{R}$ & $17.7 \pm 0.08$ & - & - & $18.2,18.3 \pm 0.01$ & $18.5^{\mathrm{c}}, 18.6 \pm 0.06^{\mathrm{d}}$ \\
\hline $\mathrm{I}(.8 \mu \mathrm{m})$ & - & - & - & $17.7,17.8 \pm 0.01$ & $18.1 \pm 0.04^{\mathrm{d}}$ \\
\hline $\mathrm{I}(.9 \mu \mathrm{m})$ & $17.2 \pm 0.10$ & - & - & - & - \\
\hline $\mathrm{J}$ & $16.4 \pm 0.10$ & $16.4 \pm 0.13$ & $16.9 \pm 0.14$ & - & - \\
\hline $\mathrm{H}$ & $15.5 \pm 0.10$ & $15.3 \pm 0.11$ & $15.9 \pm 0.16$ & - & - \\
\hline $\mathrm{K}$ & $14.4 \pm 0.05$ & $14.5 \pm 0.10$ & $14.7 \pm 0.09$ & - & - \\
\hline $\mathrm{L}$ & $12.6 \pm 0.10$ & - & - & - & $12.9 \pm 0.05^{\mathrm{e}}$ \\
\hline $4.5 \mu \mathrm{m}$ & - & - & - & - & $12.1 \pm 0.05^{\mathrm{e}}$ \\
\hline $5.7 \mu \mathrm{m}$ & - & - & - & - & $11.4 \pm 0.10^{\mathrm{e}}$ \\
\hline $8.0 \mu \mathrm{m}$ & - & - & - & - & $10.1 \pm 0.05^{\mathrm{e}}$ \\
\hline $10.2 \mu \mathrm{m}$ & $7.1 \pm 0.20$ & - & - & - & $8.3^{\mathrm{f}}$ \\
\hline $24 \mu \mathrm{m}$ & - & - & - & - & $7.5 \pm 0.10$ \\
\hline $70 \mu \mathrm{m}$ & - & - & - & - & $4.3^{\mathrm{g}}$ \\
\hline $160 \mu \mathrm{m}$ & - & - & - & - & $-2.5^{\mathrm{g}}$ \\
\hline
\end{tabular}

a2MASS, Dec., 1997

${ }^{\mathrm{b}}$ CCD photometry from Massey(2005) obtained Oct $2 \&$ 4, 2000 and Sep. 18, 2001, respectively.

${ }^{\mathrm{c}}$ From the flux-calibrated MMT spectrum

${ }^{\mathrm{d}}$ From the polarimetry frames

eSpitzer - IRAC. Here we give the mean of the four observations. The calibration uncertainties dominate the photometric errors of the individual observations; therefore the errors of the separate observations are the same. When the dispersion in the mean magnitude is less than the calibration error we quote the latter. In only one case, Ch $3(5.7 \mu \mathrm{m})$, the error of the mean was greater, $0.10 \mathrm{mag}$.

${ }^{\mathrm{f}}$ From integration of the IRS spectrum over the $\mathrm{N}$ band filter.

${ }^{\mathrm{g}}$ The upper limit in magnitudes corresponding to $13.98 \mathrm{mJy}$ and $1.5 \mathrm{Jy}$ at $70 \mu \mathrm{m}$ and $160 \mu \mathrm{m}$, respectively. The fluxes were converted to magnitudes using the Vega spectral model (Cohen 1996) and integrating over the bandpass. 
Table 3. The Strong Emission lines and Representative Absorption Lines in Variable A

\begin{tabular}{ccc}
\hline \hline Line Id. & $\begin{array}{c}\text { Hel. Vel. } \\
\mathrm{km} \mathrm{s}^{-1}\end{array}$ & $\begin{array}{c}\mathrm{W}_{\lambda} \\
\AA\end{array}$ \\
\hline
\end{tabular}

Emission

$\begin{array}{lcc}\mathrm{H} \alpha & -150.2^{\mathrm{a}} & 51.1^{\mathrm{b}}, 52.1^{\mathrm{c}} \\ \mathrm{H} \beta & -155.8^{\mathrm{d}} & 9.4^{\mathrm{b}}, 10.9^{\mathrm{c}} \\ \mathrm{H} \gamma & -147.6 & 1.9 \\ \text { Ca II } \lambda 8498 & -130.7 & 4.4 \\ \text { Ca II } \lambda 8542 & -130.4 & 6.0 \\ \text { [Ca II] } \lambda 7291 & -149.9 & 1.9 \\ \text { [Ca II] } \lambda 7324 & -170.5 & 2.5 \\ \text { K I } \lambda 7665 & -96.0 & 1.9 \\ \text { K I } \lambda 7699 & -116.5 & 3.4\end{array}$

Absorption

\begin{tabular}{lcc} 
Ca II H & -165.3 & 5.2 \\
Ca II K & -170.2 & 10.0 \\
Fe I $\lambda 4046$ & -174.1 & 1.4 \\
Fe I $\lambda 4064$ & -141.0 & 0.4 \\
Sr II $\lambda 4077$ & -140.9 & 1.3 \\
Ca I $\lambda 4226$ & -218.7 & 1.0 \\
Fe I $\lambda 4250$ & -195.0 & 0.7 \\
Fe I $\lambda 4271$ & -145.7 & 1.1 \\
Fe I $\lambda 4325$ & -140.3 & 0.8 \\
Fe I $\lambda 4383$ & -167.2 & 0.9 \\
Mg I $\lambda 5167$ & -161.2 & - \\
Na I (D) $\lambda 5890$ & -191.1 & 1.1 \\
\hline & \\
${ }^{a}$ Thomson scattering wings at -975 and $+882 \mathrm{~km} \mathrm{~s}^{-1}$ \\
${ }^{\mathrm{b}}$ without wings \\
${ }^{{ }^{c} \text { with wings }}$ \\
${ }^{\mathrm{d}}$ Thomson scattering wings at -770 and $+794 \mathrm{~km} \mathrm{~s}^{-1}$
\end{tabular}


Table 4. The Stronger Uncertain and Unidentified Emission and Absorption Lines

\begin{tabular}{|c|c|c|}
\hline$\underset{\AA}{\text { Measured } \lambda}$ & $\begin{array}{c}\text { Predicted } \lambda^{\mathrm{a}} \\
\AA\end{array}$ & Comment \\
\hline \multicolumn{3}{|l|}{ Emission } \\
\hline 4359.5 & 4361.7 & $\begin{array}{l}\text { Probable blend of [Fe II] } \lambda 4359.3 \text { and [OIII] } \lambda 4363.2 \\
\text { from nearby nebulosity }\end{array}$ \\
\hline 4921.3 & 4823.8 & $\begin{array}{l}\text { very broad feature; a blend of several lines probably } \\
\text { including Fe II } \lambda 4923.9,[\mathrm{OIII}] \lambda 4931.8 \text {, [FeII }] \lambda 4924.5\end{array}$ \\
\hline 5004.5 & 5007.0 & [OIII $] \lambda 5006.8$, possibly blended with $[\mathrm{FeII}]$ \\
\hline 5017.1 & 5019.6 & Fe II $\lambda 5018.4,[\mathrm{FeII}] \lambda 5020.2$ \\
\hline 5038.6 & 5041.1 & possibly Si II $\lambda 5041.1$ \\
\hline 5079.4 & 5081.9 & unidentified \\
\hline 5510.5 & 5513.3 & very narrow \\
\hline 6397.3 & 6400.5 & unidentified \\
\hline 6495.4 & 6498.6 & unidentified \\
\hline 7247.5 & 7251.1 & unidentified \\
\hline \multicolumn{3}{|l|}{ Absorption } \\
\hline 3823.2 & 3825.5 & unidentified \\
\hline 3855.7 & 3858.0 & unidentified \\
\hline 5057.1 & 5060.1 & unidentified \\
\hline 7236.1 & 7240.4 & unidentified \\
\hline 7273.0 & 7277.4 & unidentified \\
\hline 7337.2 & 7341.6 & unidentified \\
\hline
\end{tabular}

${ }^{a}$ Respectively determined using the mean velocity of the hydrogen emission lines $\left(-150 \mathrm{~km} \mathrm{~s}^{-1}\right)$ and the mean velocity of the absorption lines $\left(-167.6 \mathrm{~km} \mathrm{~s}^{-1}\right)$. 